\title{
Random Access for Elastic and Inelastic Traffic in WLANs
}

\author{
Man Hon Cheung, Student Member, IEEE, Amir-Hamed Mohsenian-Rad, Member, IEEE, \\ Vincent W.S. Wong, Senior Member, IEEE, and Robert Schober, Fellow, IEEE
}

\begin{abstract}
In this paper, we consider the problem of random access in wireless local area networks (WLANs) with each station generating either elastic or inelastic traffic. Elastic traffic is usually non-real-time, while inelastic traffic is usually coming from real-time applications. We formulate a network utility maximization (NUM) problem, where the optimization variables are the persistent probabilities of the stations and the utilities are either concave or sigmoidal functions. Sigmoidal utility functions can better represent inelastic traffic sources compared to concave utility functions commonly used in the existing random access literature. However, they lead to non-convex NUM problems which are not easy to solve in general. By applying the dual decomposition method, we propose a subgradient algorithm to solve the formulated NUM problem. We also develop closedform solutions for the dual subproblems involving sigmoidal functions that have to be solved in each iteration of the proposed algorithm. Furthermore, we obtain a sufficient condition on the link capacities which guarantees achieving the global optimal solution when our proposed algorithm is being used. If this condition is not satisfied, then we can still guarantee that the optimal value of the objective function is within some lower and upper bounds. We perform various simulations to validate our analytical models when the available link capacities meet or do not meet the sufficient optimality condition.
\end{abstract}

Index Terms-Wireless random access, medium access control, network utility maximization, non-convex optimization, sigmoidal function, elastic and inelastic traffic, real-time applications.

\section{INTRODUCTION}

$\mathbf{I}$ $\mathrm{N}$ a wireless network, a medium access control (MAC) protocol is used to coordinate access to the shared wireless medium for mobile stations. In general, there are two types of MAC protocols in a wireless network: scheduling-based and contention-based. Contention-based random access protocols are scalable and flexible, and are widely used in wireless local area networks (WLANs).

In this paper, we study random access in WLANs within the network utility maximization (NUM) framework. Most of the previous work in NUM-based random access (e.g., in [1]) focuses only on non-real-time applications, such as file transfer and e-mail, where the data traffic is elastic. Additionally, we consider real-time applications, such as video streaming and voice over IP services, which entail inelastic traffic.

Manuscript received on October 7, 2009; revised on February 5, 2010 and March 27, 2010; and accepted on March 29, 2010. The review of this paper was coordinated by Dr. Daniele Tarchi.

This research is funded by AUTO21, a member of the Network of Centres of Excellence of Canada program. The authors are with the Department of Electrical and Computer Engineering, The University of British Columbia, Vancouver, BC, Canada, V6T 1Z4, e-mail: \{mhcheung, hamed, vincentw, rschober\}@ece.ubc.ca.
Unlike elastic data sources that are modeled by concave utility functions, inelastic sources are modeled by non-concave utility functions, in particular sigmoidal utility functions, leading to NUM problems which are usually difficult to solve. NUM problems with sigmoidal utility functions have previously been considered in various networking design problems such as Internet congestion control [2], [3], downlink power allocation [4], power control [5], and radio resource allocation [6]. But no prior work has addressed NUM problems with sigmoidal utility functions in random access systems.

To tackle the non-convexity of of a random access NUM problem, we use the subgradient projection method. For concave utility functions, we extend the work in [1] by not restricting the utility functions to remain concave after a logarithmic change of variables, but allowing the possibilities of concave, convex, or sigmoidal utility functions. For sigmoidal utility functions, each iteration in our algorithm involves only updating the dual variables with some closedform expressions. The Karush-Kuhn-Tucker (KKT) optimality conditions of the dual problem are also derived. Moreover, we provide a sufficient condition on the wireless link capacities which guarantee our algorithm to find the exact global optimal solution of the NUM problem. If this condition is not satisfied, we can still obtain upper and lower bounds for the optimal objective value. The bounds approach each other when the duality gap is zero.

The rest of this paper is organized as follows. The system model is described in Section II. We present our centralized algorithm and the optimality conditions for the dual problem in Section III. We study the condition on capacity that results in optimal or sub-optimal solutions in Section IV. Simulation results are given in Section V. The paper is concluded in Section VI.

\section{SySTEM MODEL}

Consider a WLAN with a single access point (AP) and a set of $N$ mobile stations, denoted by $\mathcal{N}=\{1,2, \ldots, N\}$. All stations are one-hop neighbors to the AP. We only consider the uplink scenario, where each station $i \in \mathcal{N}$ can access the shared medium with a persistent probability $p_{i}$. We consider using a slotted Aloha MAC protocol, where time is divided into equal time slots. The stations attempt to access the shared channel at the beginning of each time slot according to their persistent probabilities. Notice that the choice of persistent probabilities can be transformed into equivalent contention window sizes that can be implemented directly in IEEE 802.11 WLANs [7]. 
Let $p_{i}^{\text {succ }}$ denote the probability that a transmission from station $i \in \mathcal{N}$ is successful, i.e., the transmission does not experience any collision. We have

$$
p_{i}^{\text {succ }}(\boldsymbol{p})=p_{i} \prod_{j \in \mathcal{N} \backslash\{i\}}\left(1-p_{j}\right), \quad \forall i \in \mathcal{N},
$$

where $\boldsymbol{p}=\left(p_{i}, i \in \mathcal{N}\right)$. For the rest of the paper, we will use bold symbols to denote vectors with components $\forall i \in \mathcal{N}$. Given the capacity $c_{i}$ for user $i$, the average data rate for station $i$ is $x_{i}=c_{i} p_{i}^{\text {succ }}(\boldsymbol{p})$, which is a function of both $c_{i}$ and $p$. We denote the utility function of each station $i \in \mathcal{N}$ by $U_{i}\left(x_{i}\right)$, which is a non-decreasing function in $x_{i}$. So we can control the level of satisfaction of station $i$ by tuning $x_{i}$. Each station may have either elastic or inelastic traffic. Let $\mathcal{N}_{\mathcal{E}}$ and $\mathcal{N}_{\mathcal{I}}$ denote the sets of stations with elastic and inelastic traffic, respectively. We notice that $\mathcal{N}_{\mathcal{E}} \cap \mathcal{N}_{\mathcal{I}}=\phi$ and $\mathcal{N}_{\mathcal{E}} \cup \mathcal{N}_{\mathcal{I}}=\mathcal{N}$. For each user $v \in \mathcal{N}_{\mathcal{E}}$, we can use a concave function to model the utility. A common example is the $\alpha$-fair utility function (see Fig. 1) [8]:

$$
\begin{aligned}
& U_{v}\left(x_{v}\right)= \\
& \begin{cases}\ln \left(x_{v}+1\right), & \text { if } \alpha_{v}=1, \\
\left(1-\alpha_{v}\right)^{-1}\left[\left(x_{v}+1\right)^{\left(1-\alpha_{v}\right)}-1\right], & \text { if } \alpha_{v} \in(0,1) \cup(1, \infty),\end{cases}
\end{aligned}
$$

where $\alpha_{v}$ is a fixed utility parameter.

On the other hand, for each user $w \in \mathcal{N}_{\mathcal{I}}$, the utility function depends on the quality of service (QoS) requirements of the running voice and video applications. We can use a sigmoidal utility function $U_{w}\left(x_{w}\right)$ to model these applications such that $U_{w}^{\prime \prime}\left(x_{w}\right)>0$ for $x_{w}<x_{w}^{i n}$ and $U_{w}^{\prime \prime}\left(x_{w}\right)<0$ for $x_{w}>x_{w}^{i n}$, where $x_{w}^{i n}$ is the point of inflection. In particular, we can use the sigmoidal function (see Fig. 1) defined as [9]:

$$
U_{w}\left(x_{w}\right)=\frac{x_{w}^{a_{w}}}{k_{w}+x_{w}^{a_{w}}},
$$

where $x_{w} \geq 0, a_{w}>1, k_{w}>0$, and $x_{w}^{i n}=\sqrt[a w]{\frac{k_{w}\left(a_{w}-1\right)}{a_{w}+1}}$. With the logarithmic change of variables $\bar{x}_{i} \triangleq \ln x_{i}$ and $\bar{U}_{i}\left(\bar{x}_{i}\right) \triangleq U_{i}\left(e^{\bar{x}_{i}}\right)$, the utility functions become more convex. That is, the concave part may remain concave or turn convex [1], while the convex part always remains convex. For the concave function $U_{v}\left(x_{v}\right)$ in (2), we can see that $\bar{U}_{v}\left(\bar{x}_{v}\right)$ is a sigmoidal function with point of inflection $\bar{x}_{v}^{i n}=\ln \left(\frac{1}{\alpha_{v}-1}\right)$ for $\alpha_{v}>1$, and a convex function for $0<\alpha_{v} \leq 1$. Moreover, we have

$$
\bar{U}_{w}\left(\bar{x}_{w}\right)=\frac{1}{1+e^{-\left(a_{w} \bar{x}_{w}+b_{w}\right)}},
$$

which represents a sigmoidal function in standard form with the point of inflection $\bar{x}_{w}^{i n}=-b_{w} / a_{w}$, where $k_{w}=e^{-b_{w}}$. Note that $\bar{x}_{w}^{i n} \neq \ln x_{w}^{i n}$ in general. In the sequel, we will assume that $\bar{U}_{i}\left(\bar{x}_{i}\right)$ is sigmoidal for $\bar{x}_{i}^{\min } \leq \bar{x}_{i} \leq \bar{x}_{i}^{\max }$, $\forall i \in \mathcal{N}$. We will omit the cases where $\bar{U}_{i}\left(\bar{x}_{i}\right)$ is either a convex or a concave function for brevity, because the dual problem is straightforward in these cases. It should be noted that the solution approach discussed in the following sections can be applied to concave, convex, and sigmoidal utility functions in general.

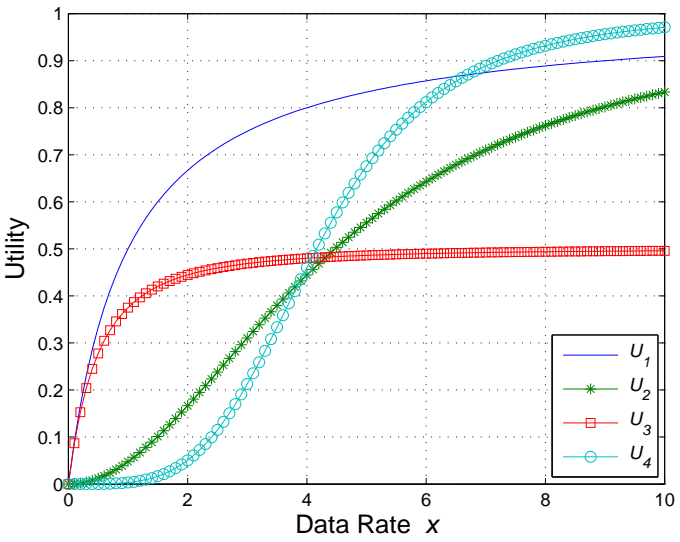

Fig. 1. Utility functions $U_{i}$ versus data rate $x$ for utility functions $U_{1}(x)=$ $1-(x+1)^{-1}, U_{2}(x)=\frac{x^{2}}{x^{2}+20}, U_{3}(x)=\frac{1}{2}\left[1-(x+1)^{-2}\right]$, and $U_{4}(x)=$ $\frac{x^{4}}{x^{4}+300}$. Notice that $U_{1}$ and $U_{3}$ are concave functions, and $U_{2}$ and $U_{4}$ are sigmoidal functions. We address both concave and sigmoidal utility functions in this paper.

\section{RANDOM ACCESS With Sigmoidal And ConcAVE UTILITIES}

\section{A. NUM for Random Access}

In this work, we consider the following NUM problem:

$$
\begin{array}{lll}
\max _{\boldsymbol{p}, \boldsymbol{x}} & \sum_{i \in \mathcal{N}} U_{i}\left(x_{i}\right)=\sum_{v \in \mathcal{N}_{\mathcal{E}}} U_{v}\left(x_{v}\right)+\sum_{w \in \mathcal{N}_{\mathcal{I}}} U_{w}\left(x_{w}\right) \\
\text { s.t. } & x_{i} \leq c_{i} p_{i}^{\text {succ }}=c_{i} p_{i} \prod_{j \in \mathcal{N} \backslash\{i\}}\left(1-p_{j}\right), & \forall i \in \mathcal{N}, \\
& x_{i}^{\text {min }} \leq x_{i} \leq x_{i}^{\text {max }}, & \forall i \in \mathcal{N}, \\
& 0 \leq p_{i} \leq 1, & \forall i \in \mathcal{N},
\end{array}
$$

where $x_{i}^{\min }$ and $x_{i}^{\max }$ are the constraints on the minimum and maximum data rates for the transmission of user $i$, respectively. Notice that problem (5) is a non-convex optimization problem, because the objective function is non-concave in general, and the first constraint is non-convex.

\section{B. Dual Method}

Using the logarithmic change of variables $\bar{x}_{i} \triangleq \ln x_{i}$, $\bar{x}_{i}^{\min } \triangleq \ln x_{i}^{\min }, \bar{x}_{i}^{\max } \triangleq \ln x_{i}^{\max }, \bar{U}_{i}\left(\bar{x}_{i}\right) \triangleq U_{i}\left(e^{\bar{x}_{i}}\right)$, and $\bar{c}_{i} \triangleq \ln c_{i}$, we can reformulate optimization problem (5) as

$$
\begin{aligned}
& \max _{\boldsymbol{p}, \bar{x}} \sum_{i \in \mathcal{N}} \bar{U}_{i}\left(\bar{x}_{i}\right)=\sum_{v \in \mathcal{N}_{\mathcal{E}}} \bar{U}_{v}\left(\bar{x}_{v}\right)+\sum_{w \in \mathcal{N}_{\mathcal{I}}} \bar{U}_{w}\left(\bar{x}_{w}\right) \\
& \text { s.t. } \quad \bar{c}_{i}+\ln p_{i}+\sum_{j \in \mathcal{N} \backslash\{i\}} \ln \left(1-p_{j}\right)-\bar{x}_{i} \geq 0, \quad \forall i \in \mathcal{N}, \\
& \bar{x}_{i}^{\text {min }} \leq \bar{x}_{i} \leq \bar{x}_{i}^{\max }, \\
& 0 \leq p_{i} \leq 1 \text {, } \\
& \forall i \in \mathcal{N}, \\
& \forall i \in \mathcal{N} \text {. }
\end{aligned}
$$

Here, the Lagrangian function is derived as

$$
\begin{aligned}
L(\boldsymbol{p}, \overline{\boldsymbol{x}}, \boldsymbol{\lambda})= & \sum_{i \in \mathcal{N}}\left(\bar{U}_{i}\left(\bar{x}_{i}\right)-\lambda_{i} \bar{x}_{i}\right) \\
& +\sum_{i \in \mathcal{N}} \lambda_{i}\left(\bar{c}_{i}+\ln p_{i}+\sum_{j \in \mathcal{N} \backslash\{i\}} \ln \left(1-p_{j}\right)\right),
\end{aligned}
$$


and the Lagrangian dual function becomes

$$
\begin{aligned}
g(\boldsymbol{\lambda})= & \sum_{i \in \mathcal{N}} \sup _{\bar{x}_{i} \in \overline{\mathcal{X}}_{i}}\left(\bar{U}_{i}\left(\bar{x}_{i}\right)-\lambda_{i} \bar{x}_{i}\right) \\
& +\sup _{\boldsymbol{p} \in \mathcal{P}} \sum_{i \in \mathcal{N}} \lambda_{i}\left(\ln p_{i}+\sum_{j \in \mathcal{N} \backslash\{i\}} \ln \left(1-p_{j}\right)\right)+\sum_{i \in \mathcal{N}} \lambda_{i} \bar{c}_{i},
\end{aligned}
$$

where $\mathcal{P}=\left\{\boldsymbol{p}: 0 \leq p_{i} \leq 1, \forall i \in \mathcal{N}\right\}$ and $\overline{\mathcal{X}}_{i}=\left\{\bar{x}_{i}:\right.$ $\left.\bar{x}_{i}^{\text {min }} \leq \bar{x}_{i} \leq \bar{x}_{i}^{\max }\right\}$. The dual problem is

$$
\begin{array}{cl}
\underset{\boldsymbol{\lambda}}{\operatorname{minimize}} & g(\boldsymbol{\lambda}) \\
\text { subject to } & \boldsymbol{\lambda} \succeq \mathbf{0} .
\end{array}
$$

In order to solve optimization problem (9), we need to solve two subproblems for each $i \in \mathcal{N}$ :

$$
\max _{\bar{x}_{i} \in \overline{\mathcal{X}}_{i}}\left(\bar{U}_{i}\left(\bar{x}_{i}\right)-\lambda_{i} \bar{x}_{i}\right)
$$

and

$$
\max _{\boldsymbol{p} \in \mathcal{P}} \sum_{i \in \mathcal{N}} \lambda_{i}\left(\ln p_{i}+\sum_{j \in \mathcal{N} \backslash\{i\}} \ln \left(1-p_{j}\right)\right) .
$$

\section{First Dual Subproblem}

To solve the first dual subproblem, we define $s_{i}\left(\bar{x}_{i}, \lambda_{i}\right)=$ $\bar{U}_{i}\left(\bar{x}_{i}\right)-\lambda_{i} \bar{x}_{i}$ and

$$
\bar{x}_{i}^{*}\left(\lambda_{i}\right)=\arg \max _{\bar{x}_{i} \in \overline{\mathcal{X}}_{i}} s_{i}\left(\bar{x}_{i}, \lambda_{i}\right) .
$$

Notice that $s_{i}$ is also a sigmoidal function in $\bar{x}_{i}$ with point of inflection $\bar{x}_{i}^{i n}$.

Lemma 1: If $\bar{x}_{i}^{\text {min }} \leq \bar{x}_{i}^{i n} \leq \bar{x}_{i}^{\max }$, we have

$$
\bar{x}_{i}^{*}\left(\lambda_{i}\right)=\arg \max _{\bar{x}_{i} \in\left\{\bar{x}_{i}^{m i n}, \bar{x}_{i}^{v}\left(\lambda_{i}\right)\right\}} s_{i}\left(\bar{x}_{i}, \lambda_{i}\right),
$$

where

$$
\bar{x}_{i}^{v}\left(\lambda_{i}\right) \triangleq \underset{\bar{x}_{i}^{i n} \leq \bar{x}_{i} \leq \bar{x}_{i}^{\max }}{\arg \max } s_{i}\left(\bar{x}_{i}, \lambda_{i}\right)
$$

Proof: It is always true that

$$
\begin{aligned}
& \max _{\bar{x}_{i} \in \overline{\mathcal{X}}_{i}} s_{i}\left(\bar{x}_{i}, \lambda_{i}\right) \\
& \quad=\max \left\{\max _{\bar{x}_{i}^{\text {min }} \leq \bar{x}_{i} \leq \bar{x}_{i}^{i n}} s_{i}\left(\bar{x}_{i}, \lambda_{i}\right), \max _{\bar{x}_{i}^{i n} \leq \bar{x}_{i} \leq \bar{x}_{i}^{\text {max }}} s_{i}\left(\bar{x}_{i}, \lambda_{i}\right)\right\} .
\end{aligned}
$$

Also notice that $s_{i}\left(\bar{x}_{i}, \lambda_{i}\right)$ is a convex function in $\bar{x}_{i}$ for $\bar{x}_{i}^{\text {min }} \leq \bar{x}_{i} \leq \bar{x}_{i}^{i n}$. Thus, we have $\arg \max _{\bar{x}_{i}^{m i n} \leq \bar{x}_{i} \leq \bar{x}_{i}^{i n}} s_{i}\left(\bar{x}_{i}, \lambda_{i}\right)=\left\{\bar{x}_{i}^{m i n}, \bar{x}_{i}^{i n}\right\}$. This concludes the proof.

Notice that problem (14) is convex. In fact, we can obtain a closed-form solution for (14) when $\bar{U}_{w}\left(\bar{x}_{w}\right)$ is as in (4):

Lemma 2: For $\bar{U}_{w}\left(\bar{x}_{w}\right)$ in (4), we have

$$
\begin{aligned}
& \bar{x}_{w}^{v}\left(\lambda_{w}\right)= \\
& \left\{\frac{\left[\frac{-\ln \left(\left(a_{w}-2 \lambda_{w}-\sqrt{a_{w}^{2}-4 a_{w} \lambda_{w}}\right) / 2 \lambda_{w}\right)-b_{w}}{a_{w}}\right]_{\bar{x}_{w}^{i n}}^{i n}, \text { if } a_{w} \geq 4 \lambda_{w},}{\bar{x}_{w}^{\text {max }},},\right.
\end{aligned}
$$

where $[z]_{w}^{y}=\min \{\max \{z, w\}, y\}$.

Proof: Since $s_{w}\left(\bar{x}_{w}, \lambda_{w}\right)$ is concave for $\bar{x}_{w}^{i n} \leq \bar{x}_{w} \leq$ $\bar{x}_{w}^{\max }$, by taking the derivative, we have

$$
\bar{U}_{w}^{\prime}\left(\bar{x}_{w}^{v}\right)-\lambda_{w}=\frac{a_{w} e^{-\left(a_{w} \bar{x}_{w}^{v}+b_{w}\right)}}{\left[1+e^{-\left(a_{w} \bar{x}_{w}^{v}+b_{w}\right)}\right]^{2}}-\lambda_{w}=0 .
$$

Let $y_{w}=e^{-\left(a_{w} \bar{x}_{w}^{v}+b_{w}\right)}$, we obtain $\lambda_{w} y_{w}^{2}+\left(2 \lambda_{w}-a_{w}\right) y_{w}+$ $\lambda_{w}=0$. We can consider two cases:

Case I: If $a_{w} \geq 4 \lambda_{w}$, since $0 \leq y_{w} \leq 1$, we can take the root $y_{w}=\frac{a_{w}-2 \lambda_{w}-\sqrt{a_{w}^{2}-4 a_{w} \lambda_{w}}}{2 \lambda_{w}}$, so

$$
\bar{x}_{w}^{v}\left(\lambda_{w}\right)=\left[\frac{-\ln \left(\left(a_{w}-2 \lambda_{w}-\sqrt{a_{w}^{2}-4 a \lambda_{w}}\right) / 2 \lambda_{w}\right)-b_{w}}{a_{w}}\right]_{\bar{x}_{w}^{i n}}^{\bar{x}_{w}^{\max }} .
$$

Case II: If $a_{w}<4 \lambda_{w}$, we have $s_{w}^{\prime}\left(\bar{x}_{w}, \lambda_{w}\right)<0$ and $s_{w}\left(\bar{x}_{w}, \lambda_{w}\right)$ is decreasing in $\bar{x}_{w}$. Thus,

$$
\bar{x}_{w}^{v}\left(\lambda_{w}\right)=\underset{\bar{x}_{w}^{i n} \leq \bar{x}_{w} \leq \bar{x}_{w}^{\max }}{\arg \max } s_{w}\left(\bar{x}_{w}, \lambda_{w}\right)=\bar{x}_{w}^{i n} .
$$

Considering the two cases above, we can fully characterize $\bar{x}_{w}^{v}\left(\lambda_{w}\right)$ as in (18) and (19).

\section{Second Dual Subproblem}

For the second dual subproblem, given $\lambda$, we have

$$
\begin{aligned}
\max _{\boldsymbol{p} \in \mathcal{P}} & \sum_{i \in \mathcal{N}} \lambda_{i}\left(\ln p_{i}+\sum_{j \in \mathcal{N} \backslash\{i\}} \ln \left(1-p_{j}\right)\right) \\
& =\sum_{i \in \mathcal{N}} \max _{0 \leq p_{i} \leq 1}\left(\lambda_{i} \ln p_{i}+\left(\sum_{j \in \mathcal{N} \backslash\{i\}} \lambda_{j}\right) \ln \left(1-p_{i}\right)\right) .
\end{aligned}
$$

Since the problem at the right hand side in (20) is convex, we can apply the first order necessary and sufficient optimization condition to obtain the given optimal solution [1] as follows

$$
p_{i}^{*}(\boldsymbol{\lambda})=\frac{\lambda_{i}}{\sum_{j \in \mathcal{N}} \lambda_{j}}, \quad \text { if } \sum_{j \in \mathcal{N}} \lambda_{j} \neq 0 .
$$

\section{E. Centralized Algorithm for Random Access}

We define

$$
\lambda_{i}^{c}=\min \left\{\lambda \geq 0: s_{i}\left(\bar{x}_{i}^{\text {min }}, \lambda\right)=\max _{\bar{x}_{i}^{i n} \leq \bar{x}_{i} \leq \bar{x}_{i}^{\text {max }}} s_{i}\left(\bar{x}_{i}, \lambda\right)\right\} .
$$

Thus, $\bar{x}_{i}^{*}\left(\lambda_{i}^{c}\right)$ has two solutions: $\bar{x}_{i}^{*}\left(\lambda_{i}^{c}\right)=\bar{x}_{i}^{\text {min }}$ and $\bar{x}_{i}^{i n} \leq$ $\bar{x}_{i}^{*}\left(\lambda_{i}^{c}\right) \leq \bar{x}_{i}^{\max }$. As shown in Fig. $2, \bar{x}_{i}^{*}\left(\lambda_{i}\right)$ is discontinuous at $\lambda_{i}^{c}$. Consider $g(\boldsymbol{\lambda})=\sup _{\boldsymbol{p} \in \mathcal{P}, \overline{\boldsymbol{x}} \in \overline{\mathcal{X}}} L(\boldsymbol{p}, \overline{\boldsymbol{x}}, \boldsymbol{\lambda})$, we apply Danskin's Theorem [10] to find the subdifferential $\partial g(\boldsymbol{\lambda})$ (i.e., the set of all subgradients of $g(\boldsymbol{\lambda})$ )

$$
\partial g(\boldsymbol{\lambda})=\operatorname{conv}\left\{\nabla_{\boldsymbol{\lambda}} L(\boldsymbol{p}, \overline{\boldsymbol{x}}, \boldsymbol{\lambda}): \boldsymbol{p} \in \boldsymbol{p}^{*}(\boldsymbol{\lambda}), \overline{\boldsymbol{x}} \in \overline{\boldsymbol{x}}^{*}(\boldsymbol{\lambda})\right\},
$$

where $\operatorname{conv}\{\mathcal{H}\}$ is the convex hull of set $\mathcal{H}$ and $\nabla_{\boldsymbol{\lambda}} L(\boldsymbol{p}, \overline{\boldsymbol{x}}, \boldsymbol{\lambda})=\left(\frac{\partial L(\boldsymbol{p}, \overline{\boldsymbol{x}}, \boldsymbol{\lambda})}{\partial \lambda_{1}}, \ldots, \frac{\partial L(\boldsymbol{p}, \overline{\boldsymbol{x}}, \boldsymbol{\lambda})}{\partial \lambda_{N}}\right)^{T}$ denotes the gradient of $L$ with respect to $\lambda$, and the notation $(\cdot)^{T}$ denotes vector transpose operator. Moreover, $\boldsymbol{p}^{*}(\boldsymbol{\lambda})$ and $\overline{\boldsymbol{x}}^{*}(\boldsymbol{\lambda})$ are the solutions of (21) and (12) at $\lambda$ for all $i \in \mathcal{N}$, respectively. 


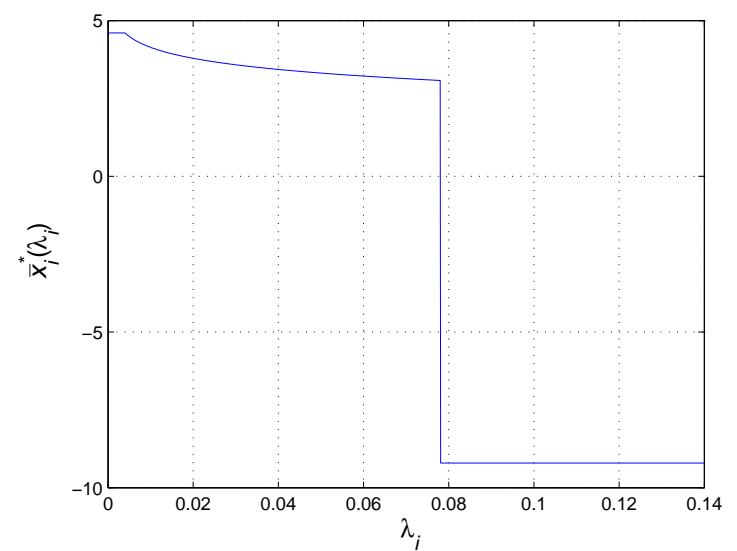

Fig. 2. The solution of the first dual subproblem $\bar{x}_{i}^{*}\left(\lambda_{i}\right)$ versus $\lambda_{i}$ for sigmoidal utility function $U_{i}(x)=\frac{x^{2}}{x^{2}+20}$. We can see that $\bar{x}_{i}^{*}\left(\lambda_{i}\right)$ is discontinuous at $\lambda_{i}=\lambda_{i}^{c}=0.0780$.

We note that $g(\boldsymbol{\lambda})$ is differentiable at $\boldsymbol{\lambda}$, if there is only one element in both sets $\boldsymbol{p}^{*}(\boldsymbol{\lambda})$ and $\overline{\boldsymbol{x}}^{*}(\boldsymbol{\lambda})$. This is always true unless $\exists i \in \mathcal{N}$ such that $\lambda_{i}=\lambda_{i}^{c}$, because in that case there are two possible solutions for $\bar{x}_{i}^{*}\left(\lambda_{i}^{c}\right)$ as discussed above. Using the subgradient projection method, we update $\left(\lambda_{i}, \forall i \in \mathcal{N}\right)$ according to the following equation:

$$
\begin{aligned}
\lambda_{i}(t+1) & =\left[\lambda_{i}(t)-\alpha(t)\left(\bar{c}_{i}+\ln p_{i}^{*}(\boldsymbol{\lambda}(t))\right.\right. \\
& \left.\left.+\sum_{j \in \mathcal{N} \backslash\{i\}} \ln \left(1-p_{j}^{*}(\boldsymbol{\lambda}(t))\right)-\bar{x}_{i}^{*}\left(\lambda_{i}(t)\right)\right)\right]^{+},
\end{aligned}
$$

where $[z]^{+}=\max \{z, 0\}$ and $t$ is the index of the iteration. With a diminishing step size $\alpha(t) \geq 0$ such that $\lim _{t \rightarrow \infty} \alpha(t)=0$ and $\sum_{t=1}^{\infty} \alpha(t)=\infty$ (e.g., we can choose $\alpha(t)=m / t$, where $m$ is a positive constant), it can be shown that $\lambda_{i}(t)$ converges to the dual optimal solution $\lambda_{i}^{*}$ as $t \rightarrow \infty$ [10]. The algorithm to solve problem (6) is shown in Algorithm 1. We will discuss the optimality of its solution in the next section.

\section{F. General Optimality Conditions}

We have the following general optimality condition for the dual problem in (9):

Theorem 1: Vector $\lambda^{*}$ is the solution of problem (9) if and only if $\lambda^{*} \succeq \mathbf{0}$ and

1) If $\lambda_{i}^{*}=0$, we have

$$
\bar{c}_{i}+\ln p_{i}^{*}\left(\boldsymbol{\lambda}^{*}\right)+\sum_{j \in \mathcal{N} \backslash\{i\}} \ln \left(1-p_{j}^{*}\left(\boldsymbol{\lambda}^{*}\right)\right)-\bar{x}_{i}^{v}\left(\lambda_{i}^{*}\right) \geq 0 .
$$

2) If $\lambda_{i}^{*}>0$ and $\lambda_{i}^{*} \neq \lambda_{i}^{c}$, we have

$$
\bar{c}_{i}+\ln p_{i}^{*}\left(\boldsymbol{\lambda}^{*}\right)+\sum_{j \in \mathcal{N} \backslash\{i\}} \ln \left(1-p_{j}^{*}\left(\boldsymbol{\lambda}^{*}\right)\right)-\bar{x}_{i}^{v}\left(\lambda_{i}^{*}\right)=0 .
$$

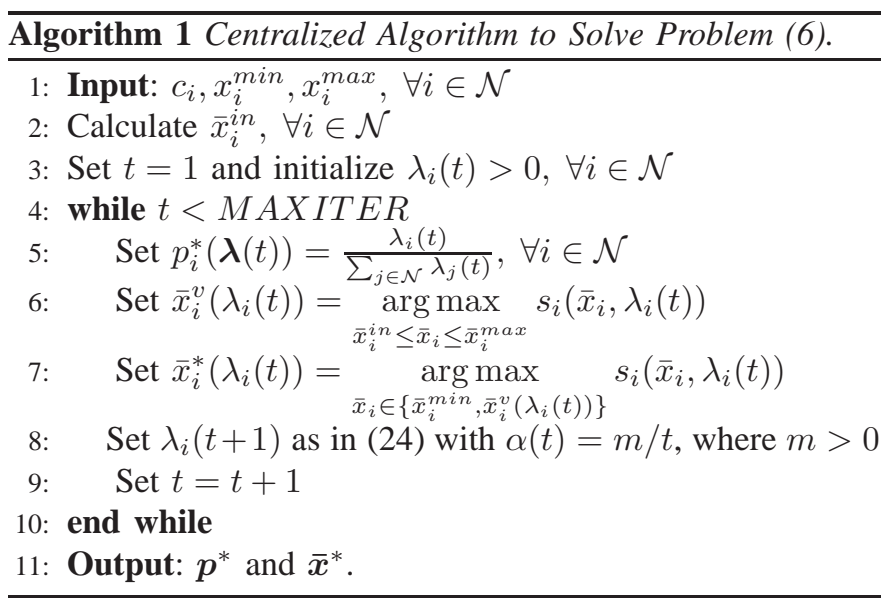

3) If $\lambda_{i}^{*}>0$ and $\lambda_{i}^{*}=\lambda_{i}^{c}$, we have

$$
\bar{c}_{i}+\ln p_{i}^{*}\left(\boldsymbol{\lambda}^{*}\right)+\sum_{j \in \mathcal{N} \backslash\{i\}} \ln \left(1-p_{j}^{*}\left(\boldsymbol{\lambda}^{*}\right)\right)-\bar{x}_{i}^{v}\left(\lambda_{i}^{*}\right) \leq 0,
$$

and

$$
\bar{c}_{i}+\ln p_{i}^{*}\left(\boldsymbol{\lambda}^{*}\right)+\sum_{j \in \mathcal{N} \backslash\{i\}} \ln \left(1-p_{j}^{*}\left(\boldsymbol{\lambda}^{*}\right)\right)-\bar{x}_{i}^{\text {min }} \geq 0 .
$$

Proof: First, for primal feasibility, we have $\boldsymbol{\lambda}^{*} \succeq \mathbf{0}$. For cases 1 and $2, g(\boldsymbol{\lambda})_{i}$ is differentiable at $\lambda_{i}=\lambda_{i}^{*}$, where $g(\boldsymbol{\lambda})_{i}$ is the $i^{\text {th }}$ element in $g(\boldsymbol{\lambda})$. The $i^{\text {th }}$ entry of the derivative becomes

$\nabla g\left(\boldsymbol{\lambda}^{*}\right)_{i}=\bar{c}_{i}+\ln p_{i}^{*}\left(\boldsymbol{\lambda}^{*}\right)+\sum_{j \in \mathcal{N} \backslash\{i\}} \ln \left(1-p_{j}^{*}\left(\boldsymbol{\lambda}^{*}\right)\right)-\bar{x}_{i}^{v}\left(\lambda_{i}^{*}\right)$.

Since problem (9) is convex, the result directly follows from [11, pp. 142]. For case $3, g(\boldsymbol{\lambda})_{i}$ is non-differentiable at $\lambda_{i}=$ $\lambda_{i}^{*}$; therefore, the KKT condition is $0 \in \partial g\left(\boldsymbol{\lambda}^{*}\right)_{i}$. From (23), we have

$$
\begin{array}{r}
\partial g\left(\boldsymbol{\lambda}^{*}\right)_{i} \\
=\operatorname{conv}\left\{\bar{c}_{i}+\ln p_{i}^{*}\left(\boldsymbol{\lambda}^{*}\right)+\sum_{j \in \mathcal{N} \backslash\{i\}} \ln \left(1-p_{j}^{*}\left(\boldsymbol{\lambda}^{*}\right)\right)-\bar{x}_{i}^{*}:\right. \\
\left.\bar{x}_{i}^{*} \in \bar{x}_{i}^{*}\left(\lambda_{i}^{*}\right)\right\} \\
=\left[\bar{c}_{i}+\ln p_{i}^{*}\left(\boldsymbol{\lambda}^{*}\right)+\sum_{j \in \mathcal{N} \backslash\{i\}} \ln \left(1-p_{j}^{*}\left(\boldsymbol{\lambda}^{*}\right)\right)-\bar{x}_{i}^{v}\left(\lambda_{i}^{*}\right),\right. \\
\left.\bar{c}_{i}+\ln p_{i}^{*}\left(\boldsymbol{\lambda}^{*}\right)+\sum_{j \in \mathcal{N} \backslash\{i\}} \ln \left(1-p_{j}^{*}\left(\boldsymbol{\lambda}^{*}\right)\right)-\bar{x}_{i}^{\text {min }}\right],
\end{array}
$$

which is simply an interval. Considering the lower and upper bounds in the interval, we can directly derive (27) and (28), respectively.

Since the dual problem is convex, the KKT conditions (25)(28) are necessary and sufficient [11, p. 139]. By using the following theorem, we can determine whether Algorithm 1 can solve the NUM problem.

Theorem 2: If $\lambda_{i}^{*} \neq \lambda_{i}^{c}, \forall i \in \mathcal{N}$, then Algorithm 1 finds the optimal solution of problem (6). 
Proof: If $\lambda_{i}^{*} \neq \lambda_{i}^{c}, \forall i \in \mathcal{N}$, from the discussion in Section III-E, $\boldsymbol{p}^{*}=\boldsymbol{p}^{*}\left(\boldsymbol{\lambda}^{*}\right)$ and $\overline{\boldsymbol{x}}^{*}=\overline{\boldsymbol{x}}^{*}\left(\boldsymbol{\lambda}^{*}\right)$ form the unique minimizer of Lagrangian $L\left(\boldsymbol{p}, \overline{\boldsymbol{x}}, \boldsymbol{\lambda}^{*}\right)$. Thus $g(\boldsymbol{\lambda})$ is differentiable at $\lambda^{*}$ by (23). Finally, from [12, Property 6.5(c)], the primal problem (6) has a saddle point $\left(\boldsymbol{p}^{*}, \overline{\boldsymbol{x}}^{*}, \boldsymbol{\lambda}^{*}\right)$. By [12, Theorem 5.3], $\boldsymbol{p}^{*}$ and $\overline{\boldsymbol{x}}^{*}$ are the global optimum of the primal problem (6).

\section{Optimality AND SUb-OPTIMALITY}

In this section, we assume that $x_{i}^{\max }=c_{i}, \forall i \in \mathcal{N}$ such that the optimal value of the objective function is restricted by capacity $\boldsymbol{c}=\left(c_{i}, i \in \mathcal{N}\right)$ only, but not the data rate bound $\boldsymbol{x}^{\max }=\left(x_{i}^{\max }, i \in \mathcal{N}\right)$. Next, we discuss certain conditions on vector $c$ which affect optimality and sub-optimality of Algorithm 1.

\section{A. Optimal Solution}

We first provide a sufficient condition on the link capacities for optimality of Algorithm 1:

Theorem 3: With $x_{i}^{\max }=\infty$, suppose $\lambda_{i}^{c}$ and $\bar{x}_{i}^{v}\left(\lambda_{i}^{c}\right)$ are obtained by (22) and (14) for any $i \in \mathcal{N}$, respectively. We define

$$
c_{i}^{c}=\frac{e^{\bar{x}_{i}^{v}\left(\lambda_{i}^{c}\right)}}{p_{i}^{*}\left(\boldsymbol{\lambda}^{c}\right) \prod_{j \in \mathcal{N} \backslash\{i\}}\left(1-p_{j}^{*}\left(\boldsymbol{\lambda}^{c}\right)\right)}, \quad \forall i \in \mathcal{N},
$$

where $p_{i}^{*}\left(\boldsymbol{\lambda}^{c}\right)$ is as in (21). Here, $\boldsymbol{c}^{c}$ denotes the vector of critical link capacities. If $\boldsymbol{c} \succ \boldsymbol{c}^{c}$, then Algorithm 1 can obtain the optimal solution in problem (6), and thus that of problem (5).

Proof: Let $\lambda^{*}$ be the dual optimal solution and assume that $c \succ \boldsymbol{c}^{c}$. From (31), we have $\bar{c}_{i}+\ln p_{i}^{*}\left(\boldsymbol{\lambda}^{c}\right)+$ $\sum_{j \in \mathcal{N} \backslash\{i\}} \ln \left(1-p_{j}^{*}\left(\boldsymbol{\lambda}^{c}\right)\right)-\bar{x}_{i}^{v}\left(\lambda_{i}^{c}\right)>0$, for any $i \in \mathcal{N}$. We can further show that

$$
\begin{aligned}
0 \notin \partial g\left(\boldsymbol{\lambda}^{c}\right)_{i} & \\
= & {\left[\bar{c}_{i}+\ln p_{i}^{*}\left(\boldsymbol{\lambda}^{c}\right)+\sum_{j \in \mathcal{N} \backslash\{i\}} \ln \left(1-p_{j}^{*}\left(\boldsymbol{\lambda}^{c}\right)\right)-\bar{x}_{i}^{v}\left(\lambda_{i}^{c}\right),\right.} \\
& \left.\bar{c}_{i}+\ln p_{i}^{*}\left(\boldsymbol{\lambda}^{c}\right)+\sum_{j \in \mathcal{N} \backslash\{i\}} \ln \left(1-p_{j}^{*}\left(\boldsymbol{\lambda}^{c}\right)\right)-\bar{x}_{i}^{m i n}\right] .
\end{aligned}
$$

Thus, $\lambda_{i}^{*} \neq \lambda_{i}^{c}, \forall i \in \mathcal{N}$. By Theorem 2, Algorithm 1 finds the optimum of problem (5).

The key idea in the proof is that if $c \succ c^{c}, \lambda_{i}^{*}<\lambda_{i}^{c}, \forall i \in$ $\mathcal{N}$, then the optimality of the solution directly results from Theorem 2.

\section{B. Sub-optimal Solution: Upper and Lower Bounds}

Next, assume that $c \preceq c^{c}$. In this case, Algorithm 1 may only obtain a sub-optimal solution. For cases other than $c \succ$ $\boldsymbol{c}^{c}$ and $\boldsymbol{c} \preceq \boldsymbol{c}^{c}$, Algorithm 1 may obtain an optimal or suboptimal solution depending on the exact scenario. Notice that $\overline{\boldsymbol{x}}^{*}(\boldsymbol{\lambda})$ and $\boldsymbol{p}^{*}(\boldsymbol{\lambda})$ obtained from lines 5 to 7 of Algorithm 1 always satisfy the second and third constraints in problem (6), respectively. By Theorem 1, the first constraint can be satisfied in all the three cases in (25), (26), and (28). That is, for the case $\lambda_{i}^{*}>0$ and $\lambda_{i}^{*}=\lambda_{i}^{c}$, we will choose $\bar{x}_{i}^{*}\left(\lambda_{i}\right)=\bar{x}_{i}^{m i n}$. By weak duality [11, pp.225], we can obtain an upper bound for the objective value of problems (5) and (6) as

$$
\sum_{i \in \mathcal{N}} U_{i}\left(x_{i}^{*}\right)=\sum_{i \in \mathcal{N}} \bar{U}_{i}\left(\bar{x}_{i}^{*}\right) \leq g\left(\boldsymbol{\lambda}^{*}\right)=L\left(\boldsymbol{p}^{*}\left(\boldsymbol{\lambda}^{*}\right), \overline{\boldsymbol{x}}^{*}\left(\boldsymbol{\lambda}^{*}\right), \boldsymbol{\lambda}^{*}\right) .
$$

The first equality is due to the fact that problems (5) and (6) have the same objective function. The inequality is due to weak duality, and the last equality is by definition. In some cases, we can also obtain a lower bound for problem (5). If $x_{i}=$ $c_{i} p_{i}^{*}\left(\boldsymbol{\lambda}^{*}\right) \prod_{j \in \mathcal{N} \backslash\{i\}}\left(1-p_{j}^{*}\left(\boldsymbol{\lambda}^{*}\right)\right)$ satisfies constraint $x_{i}^{\text {min }} \leq$ $x_{i} \leq x_{i}^{\max }, \forall i \in \mathcal{N}$, by the optimality of $\boldsymbol{x}^{*}$, we can obtain a lower bound as

$$
\begin{aligned}
\sum_{i \in \mathcal{N}} \bar{U}_{i}\left(\bar{x}_{i}^{*}\right) & =\sum_{i \in \mathcal{N}} U_{i}\left(x_{i}^{*}\right) \\
& \geq \sum_{i \in \mathcal{N}} U_{i}\left(c_{i} p_{i}^{*}\left(\boldsymbol{\lambda}^{*}\right) \prod_{j \in \mathcal{N} \backslash\{i\}}\left(1-p_{j}^{*}\left(\boldsymbol{\lambda}^{*}\right)\right)\right) .
\end{aligned}
$$

It should be noted that both the upper and lower bounds are constructed from the same $\boldsymbol{p}^{*}\left(\boldsymbol{\lambda}^{*}\right)$ and $\overline{\boldsymbol{x}}^{*}\left(\boldsymbol{\lambda}^{*}\right)$ obtained from Algorithm 1. When the duality gap is zero, the upper and lower bounds are both equal to the optimal value of the objective function $\sum_{i \in \mathcal{N}} U_{i}\left(x_{i}^{*}\right)$.

\section{Performance Evaluation}

In this section, we consider both cases where $c \succ c^{c}$ and $c \preceq c^{c}$ for Algorithm 1. We choose $x_{i}^{\text {min }}=0.0001$ and $x_{i}^{\max }=c_{i}$, for $\forall i \in \mathcal{N}$. Here, we assume that there are two types of utility functions used in the network: a concave (Type 1) function $U_{1}\left(x_{1}\right)=1-\left(x_{1}+1\right)^{-1}$, and a sigmoidal (Type 2) function $U_{2}\left(x_{2}\right)=\frac{x_{2}^{2}}{x_{2}^{2}+20}$, as shown in Fig. 1. We can then obtain $\lambda_{1}^{c}=0.0789$ and $\lambda_{2}^{c}=0.0780$ numerically (e.g., using MATLAB as in Fig. 2). We assume that there are $N$ stations in the network, where half of them are Type 1, and the other half are Type 2 . We plot $c_{1}^{c}$ and $c_{2}^{c}$ versus the total number of stations $N$ in Fig. 3. We can see that only a linear increase in $c_{1}^{c}$ and $c_{2}^{c}$ is required for Algorithm 1 to find the optimal solution when $N$ increases.

Next, we plot the aggregate utility versus the number of stations in Fig. 4 to verify the optimality of Algorithm 1 when $c \succ c^{c}$. We can see that the result of the exhaustive search is identical to that of Algorithm 1, meaning that Algorithm 1 obtains the optimal solution. Then, we consider the case where $c \prec c^{c}$ by using the capacities $c=0.5 c^{c}$. Fig. 5 shows the upper and lower bounds obtained from Algorithm 1. Next, we focus on the case when $N=2, c_{1}=21 \mathrm{kbps}$ and $c_{2}=44 \mathrm{kbps}$ to study the resource allocation when $\boldsymbol{c} \prec \boldsymbol{c}^{c}$. With the use of diminishing step size $\alpha(t)=0.01 / t$, the allocations of persistent probabilities converge, as shown in Fig. 6. Moreover, we have noticed in the simulation that $\lambda_{1}(t) \rightarrow \lambda_{1}^{*}=\lambda_{1}^{c}$ and $\lambda_{2}(t) \rightarrow \lambda_{2}^{*}=\lambda_{2}^{c}$ as $t \rightarrow \infty$. It can be verified, by simulation, that the use of a constant step size leads to oscillatory behaviour in the dual variables and the allocation of persistent probabilities. 


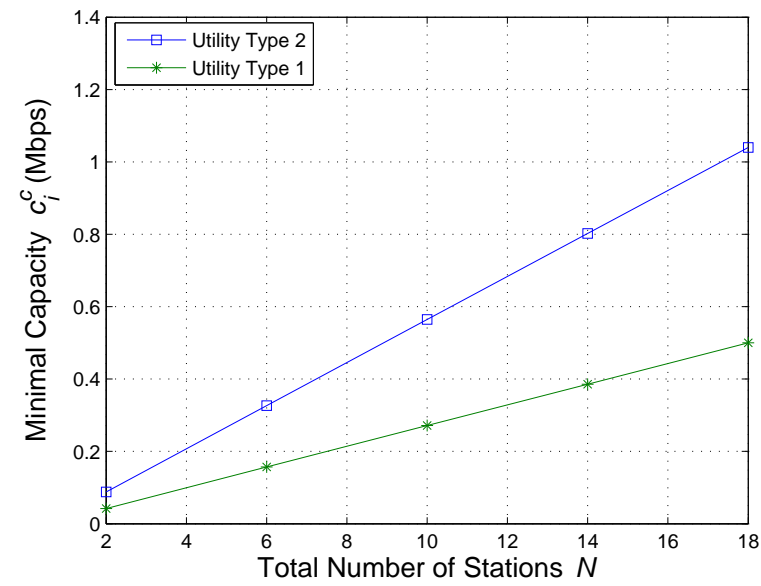

Fig. 3. The minimal capacities $c_{1}^{c}$ and $c_{2}^{c}$ for two types of utility functions versus the total number of stations $N$. Type 1 utility functions are concave, while Type 2 utility functions are sigmoidal.

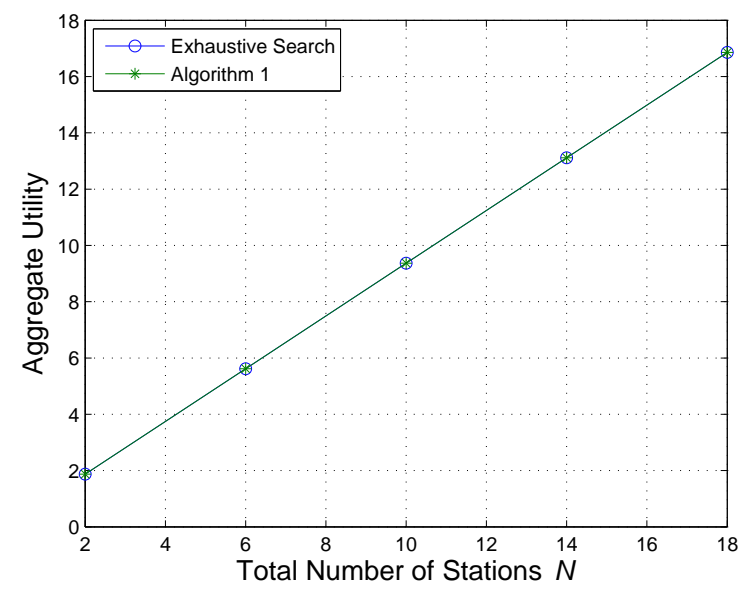

Fig. 4. Aggregate utility versus the total number of stations $N$ when $c \succ c^{c}$ using exhaustive search and Algorithm 1.

\section{Vi. CONClusions}

In this work, we proposed a random access algorithm based on the NUM framework for stations with either concave or sigmoidal utilities. We applied the dual method to solve our problem. A sufficient condition on link capacities that guarantee the optimality of the solution is proposed. Simulations have been performed to verify our analytical results.

\section{REFERENCES}

[1] J. Lee, M. Chiang, and A. R. Calderbank, "Utility-optimal randomaccess control," IEEE Trans. on Wireless Communications, vol. 6, no. 7, pp. 2741-2751, July 2007.

[2] J. Lee, R. R. Mazumdar, and N. B. Shroff, "Nonconvexity issues for internet rate control with multiclass services: Stability and optimality," in Proc. of IEEE INFOCOM, Hong Kong, China, Mar. 2004.

[3] P. Hande, S. Zhang, and M. Chiang, "Distributed rate allocation for inelastic flows," IEEE/ACM Trans. on Networking, vol. 15, no. 6, pp. 1240-1253, Dec. 2007.

[4] J. Lee, R. R. Mazumdar, and N. B. Shroff, "Downlink power allocation for multi-class wireless systems," IEEE/ACM Trans. on Networking, vol. 13 , no. 4 , pp. $854-867,2005$.

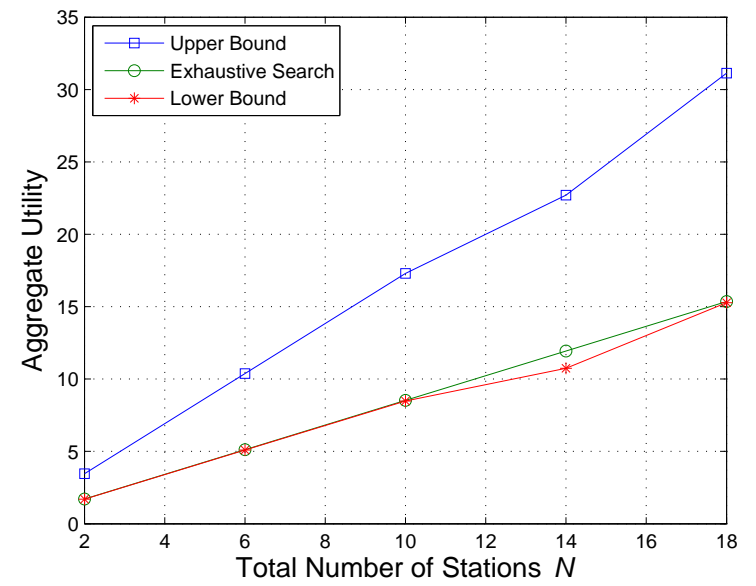

Fig. 5. Aggregate utility versus the total number of stations $N$ when $c \prec c^{c}$ using exhaustive search and Algorithm 1. The lower and upper bounds are obtained by replacing $\boldsymbol{p}^{*}\left(\boldsymbol{\lambda}^{*}\right)$ and $\overline{\boldsymbol{x}}^{*}\left(\boldsymbol{\lambda}^{*}\right)$ (i.e., the results from Algorithm 1) into the expressions in (34) and (33), respectively. We can see that the lower bound is very tight in this case. In fact, except for the case with 14 stations, the lower bound exactly matches the global optimal solution in all other considered cases.

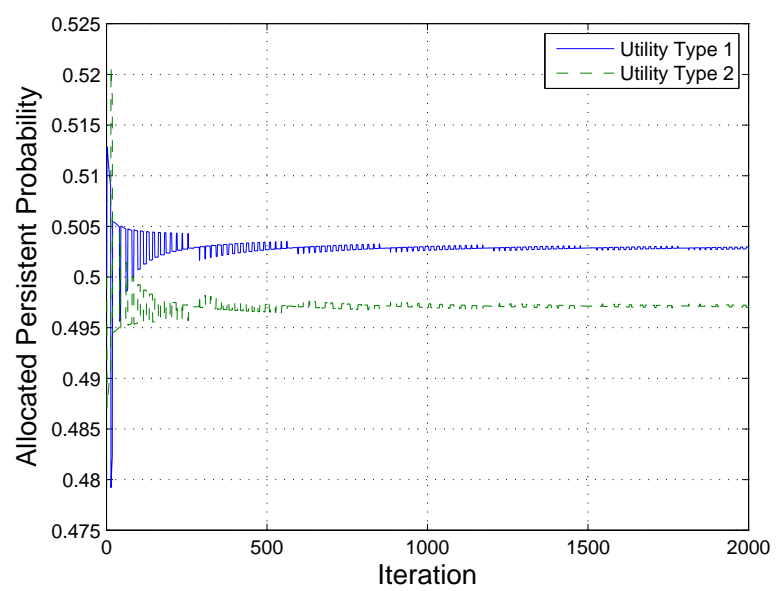

Fig. 6. Convergence of the allocation of the persistent probabilities with insufficient capacity $\boldsymbol{c} \prec \boldsymbol{c}^{c}$ using diminishing step size $\alpha(t)=0.01 / t$, even though the allocation may not be globally optimal as discussed in Section IV.

[5] M. Xiao, N. B. Shroff, and E. K. P. Chong, "A utility-based powercontrol scheme in wireless cellular systems," IEEE/ACM Trans. on Networking, vol. 11, no. 2, pp. 210-221, Apr. 2003.

[6] W.-H. Kuo and W. Liao, "Utility-based radio resource allocation for QoS traffic in wireless networks," IEEE Trans. on Wireless Commun., vol. 7, no. 7, pp. 2714-2722, July 2008.

[7] J. Lee, A. Tang, J. Huang, M. Chiang, and A. R. Calderbank, "Reverseengineering MAC: A non-cooperative game model," IEEE J. Select. Areas Commun., vol. 25, no. 6, pp. 1135-1147, Aug. 2007.

[8] J. Mo and J. Walrand, "Fair end-to-end window-based congestion control," IEEE/ACM Trans. on Networking, vol. 8, no. 5, pp. 556-567, Oct. 2000.

[9] W. G. Bardsley and R. M. W. Wood, "Critical points and sigmoidicity of positive rational functions," The American Mathematical Monthly, vol. 92, no. 1, pp. 37-48, 1985.

[10] D. P. Bertsekas, Nonlinear Programming, 2nd ed. Athena Scientific, 1999.

[11] S. Boyd and L. Vandenberghe, Convex Optimization. Cambridge, UK: Cambridge University Press, 2004.

[12] M. Minoux, Mathematical Programming: Theory and Algorithms. New York, NY: Wiley, 1986. 